\title{
A Predictive HQSAR Model for a Series of Tricycle Core Containing MMP-12 Inhibitors with Dibenzofuran Ring
}

\author{
Jamal Shamsara ${ }^{1}$ and Ahmad Shahir-Sadr ${ }^{2}$ \\ ${ }^{1}$ Pharmaceutical Research Center, School of Pharmacy, Mashhad University of Medical Sciences, Mashhad 91775-1365, Iran \\ ${ }^{2}$ Molecular and Cellular Biology Research Center, School of Medicine, Sabzevar University of Medical Sciences, \\ Sabzevar 96138-73136, Iran
}

Correspondence should be addressed to Jamal Shamsara; shamsaraj@mums.ac.ir

Received 24 August 2014; Revised 26 October 2014; Accepted 5 November 2014; Published 7 December 2014

Academic Editor: Rosaria Volpini

Copyright (C) 2014 J. Shamsara and A. Shahir-Sadr. This is an open access article distributed under the Creative Commons Attribution License, which permits unrestricted use, distribution, and reproduction in any medium, provided the original work is properly cited.

\begin{abstract}
MMP-12 is a member of matrix metalloproteinases (MMPs) family involved in pathogenesis of some inflammatory based diseases. Design of selective matrix MMPs inhibitors is still challenging because of binding pocket similarities among MMPs family. We tried to generate a HQSAR (hologram quantitative structure activity relationship) model for a series of MMP-12 inhibitors. Compounds in the series of inhibitors with reported biological activity against MMP-12 were used to construct a predictive HQSAR model for their inhibitory activity against MMP-12. The HQSAR model had statistically excellent properties and possessed good predictive ability for test set compounds. The HQSAR model was obtained for the 26 training set compounds showing cross-validated $q^{2}$ value of 0.697 and conventional $r^{2}$ value of 0.986 . The model was then externally validated using a test set of 9 compounds and the predicted values were in good agreement with the experimental results $\left(r_{\text {pred }}^{2}=0.8733\right)$. Then, the external validity of the model was confirmed by Golbraikh-Tropsha and $r_{m}^{2}$ metrics. The color code analysis based on the obtained HQSAR model provided useful insights into the structural features of the training set for their bioactivity against MMP-12 and was useful for the design of some new not yet synthesized MMP-12 inhibitors.
\end{abstract}

\section{Introduction}

Matrix metalloproteinases (MMPs) family enzymes can degrade extracellular matrix components by their proteolytic activity which depends on catalytic zinc ion [1]. The main role of macrophage metalloelastase (MMP-12) is degradation of elastin. Furthermore, MMP-12 is an interesting therapeutic target overexpressed in inflammatory pathological conditions (such as respiratory system diseases including asthma and chronic obstructive pulmonary disorder (COPD)) [2]. Effectiveness of MMP-12 inhibitors in reducing inflammation in respiratory system has been shown $[3,4]$.

The active site is highly conserved among MMPs with the exception of a loop region called $\mathrm{S1}^{\prime}$. $\mathrm{S1}^{\prime}$ pocket in MMPs active sites varies slightly among MMPs in both sequence and structure [5]. Despite available structural information, still the lack of selectivity remains as a main challenge for successfulness of MMPs inhibitors in clinical trials. Furthermore, intrinsic flexibility of MMPs active sites makes MMPs active site analysis more complicated $[6,7]$. Therefore, in this study, a ligand based approach was used to modify the side chain in a series of MMP-12 inhibitors. HQSAR (hologram quantitative structure activity relationship) is a method for QSAR (quantitative structure activity relationship) studies whose reliability has been established [8]. In the present study, a HQSAR study on a series of tricycle cores containing MMP-12 inhibitors was carried out.

\section{Methods}

2.1. Obtaining Biological Data and Generation of Molecular Structures. The structures of $35 \mathrm{MMP}-12$ inhibitors and their biological activities for inhibition of MMP-12 were taken from the literatures (Figure 1 and Table 1) $[9,10]$. As the 
TABLE 1: Actual and predicted activities of the training and test sets based on the HQSAR model. Activities were shown as pIC ${ }_{50}(\mu \mathrm{M})$.

\begin{tabular}{|c|c|c|c|c|c|}
\hline Name & $\mathrm{R}$ & Actual $\mathrm{pIC}_{50}$ values & Predicted $\mathrm{pIC}_{50}$ values & Residues & $\begin{array}{c}\text { Normalized mean } \\
\text { distance score } \\
\end{array}$ \\
\hline 10 & & 2.699 & 2.594 & 0.105 & 0.066 \\
\hline 11 & & 1.8861 & 2.05 & -0.1639 & 0.028 \\
\hline 12 & & 1.8239 & 2.144 & -0.3201 & 0.022 \\
\hline 13 & & 3.1549 & 2.688 & 0.4669 & 0.049 \\
\hline 14 & & 1.6383 & 1.646 & -0.0077 & 0.332 \\
\hline $15^{\mathrm{a}}$ & & 1.7447 & 1.754 & -0.0093 & 0.065 \\
\hline 16 & & 2.6576 & 2.672 & -0.0144 & 0.208 \\
\hline 19 & & 3.3979 & 3.706 & -0.3081 & 0.037 \\
\hline 20 & & 4 & 4.032 & -0.032 & 0.043 \\
\hline 21 & & 4 & 3.778 & 0.222 & 0.03 \\
\hline 22 & & 3.699 & 3.647 & 0.052 & 0.033 \\
\hline 23 & & 3.699 & 3.752 & -0.053 & 0.031 \\
\hline 24 & & 3 & 3.049 & -0.049 & 0.005 \\
\hline
\end{tabular}


TABLe 1: Continued.

\begin{tabular}{|c|c|c|c|c|c|}
\hline Name & $\mathrm{R}$ & Actual pIC $_{50}$ values & Predicted $\mathrm{pIC}_{50}$ values & Residues & $\begin{array}{c}\text { Normalized mean } \\
\text { distance score }\end{array}$ \\
\hline $25^{\mathrm{a}}$ & & 3.3979 & 3.17 & 0.2279 & 0.085 \\
\hline 26 & & 3 & 2.945 & 0.055 & 0.009 \\
\hline 27 & & 2.9208 & 2.949 & -0.0282 & 0.008 \\
\hline 33 & Methyl & 2.0655 & 2.341 & -0.2755 & 0 \\
\hline 34 & Ethyl & 2.5376 & 2.452 & 0.0856 & 0.01 \\
\hline 35 & i-Propyl & 2.3468 & 2.423 & -0.0762 & 0.087 \\
\hline 36 & t-Butyl & 1.7696 & 1.839 & -0.0694 & 0.554 \\
\hline 37 & i-Butyl & 2.2676 & 2.203 & 0.0646 & 0.284 \\
\hline 38 & $\mathrm{CH} 2 \mathrm{OCH} 3$ & 2.7212 & 2.571 & 0.1502 & 0.007 \\
\hline 39 & CF3 & 2.6576 & 2.543 & 0.1146 & 0 \\
\hline 40 & Cyclopropyl & 2.7959 & 2.767 & 0.0289 & 0.08 \\
\hline 41 & Cyclobutyl & 2.6383 & 2.689 & -0.0507 & 0.377 \\
\hline 42 & Cyclohexyl & 2.1427 & 2.126 & 0.0167 & 1 \\
\hline 43 & Phenyl & 2.3979 & 2.561 & -0.1631 & 0.116 \\
\hline 44 & & 3.5229 & 3.491 & 0.0319 & 0.186 \\
\hline $51^{\mathrm{a}}$ & & 2.5441 & 2.483 & 0.0611 & 0.059 \\
\hline $52^{\mathrm{a}}$ & & 2.0969 & 2.502 & -0.4051 & 0.088 \\
\hline $53^{\mathrm{a}}$ & & 2.173 & 2.146 & 0.027 & 0.297 \\
\hline $54^{\mathrm{a}}$ & & 2.5229 & 2.526 & -0.0031 & 0.049 \\
\hline $55^{\mathrm{a}}$ & & 2.1461 & 2.305 & -0.1589 & 0.324 \\
\hline
\end{tabular}


TABle 1: Continued.

\begin{tabular}{llcc}
\hline Name & Actual $\mathrm{pIC}_{50}$ values & Predicted $\mathrm{pIC}_{50}$ values & $\begin{array}{c}\text { Residues } \\
\text { Normalized mean } \\
\text { distance score }\end{array}$ \\
\hline $56^{\mathrm{a}}$ & & 2.616 & \\
0
\end{tabular}

${ }^{\mathrm{a}}$ Test set compounds.<smiles>[R]c1ccc2oc3cc(S(=O)(=O)N[C@H](C(=O)O)C(C)C)ccc3c2c1</smiles>

FIgURE 1: General structure for dataset.

activity of compound 10 is determined in both studies, we normalized the $\mathrm{IC}_{50}$ based on the reported activity for compound 10 . The range of $\mathrm{pIC}_{50}(\mu \mathrm{M})$ values for MMP-12 spans around three orders of magnitude $(\min =1.6383, \max =$ 4 ) in training set. The compounds were divided into two sets, training $(n=26)$ and test $(n=9)$ sets, according to the maintaining of structural diversity and the uniform distribution of $\mathrm{IC}_{50}$. The $\mathrm{pIC}_{50}\left(-\log \mathrm{IC}_{50}\right)$ was employed as dependent variable instead of $\mathrm{IC}_{50}$. The molecular structures were built using PyMOL (http://www.pymol.org/, The PyMOL Molecular Graphics System, Version 1.2r3pre, Schrödinger, LLC). The HQSAR model was developed by SYBYL-X1.2 molecular modeling package (Tripos International, St. Louis).

2.2. HQSAR Model Generation and Validation. HQSAR technique explores the contribution of each fragment of each molecule under study to the biological activity. As inputs, it needs datasets with their corresponding inhibitory activity in terms of $\mathrm{pIC}_{50}$. Structures in the dataset were fragmented and hashed into array bins. Molecular hologram fingerprints were then generated. Hologram was constructed by cutting the fingerprint into strings at various hologram length parameters.

After generation of descriptors, partial least square (PLS) methodology was used to find the possible correlation between dependent variable $\left(-\mathrm{pIC}_{50}\right)$ and independent variable (descriptors generated by HQSAR structural features). LOO (leave-one-out) cross-validation method was used to determine the predictive value of the model. Optimum number of components was found out using results from LOO calculations. At this step, $q^{2}$ and standard error obtained from leave-one-out cross-validation roughly estimate the predictive ability of the model. This cross-validated analysis was followed by a non-cross-validated analysis with the calculated optimum number of principle components. Conventional correlation coefficient $r^{2}$ and standard error of estimate (SEE) indicated the validity of the model. The internal validity of the model was also tested by $Y$-randomization method [11]. In this test, the dependent variables are randomly shuffled while the independent variables (descriptors) are kept unchanged. It is expected that $q^{2}$ and $r^{2}$ calculated for these random datasets will be low. Finally, a set of compounds (which were not present in model development process) with available observed activity were used for external validation of the generated model. Predictive $r^{2}\left(r_{\text {pred }}^{2}\right)$ value was calculated using

$$
r_{\text {pred }}^{2}=1-\frac{\text { PRESS }}{\mathrm{SD}}
$$

PRESS: sum of the squared deviation between predicted and actual $\mathrm{pIC}_{50}$ for the test set compounds;

SD: sum of the squared deviation between the actual $\mathrm{pIC}_{50}$ values of the compounds from the test set and the mean $\mathrm{pIC}_{50}$ value of the training set compounds.

The external validity of the model was also evaluated by Golbraikh-Tropsha [12] method and $r_{m}^{2}$ [13] metrics. For an acceptable QSAR model, the value of "average $r_{m}^{2}$ " should be $>0.5$ and "delta $r_{m}^{2}$ " should be $<0.2$. The applicability domain of the generated model was evaluated for both test and prediction sets by Euclidean based method. It calculates a normalized mean distance score for each compound in training set in range of 0 (least diverse) to 1 (most diverse). Then, it calculates the normalized mean distance score for compounds in an external set. If a score is outside the 0 to 1 range, it will be considered outside of the applicability domain. The external validity tests (Golbraikh-Tropsha and $\mathrm{Rm}^{2}$ ) and applicability domain test were done using tools available at http://dtclab.webs.com/software-tools. 


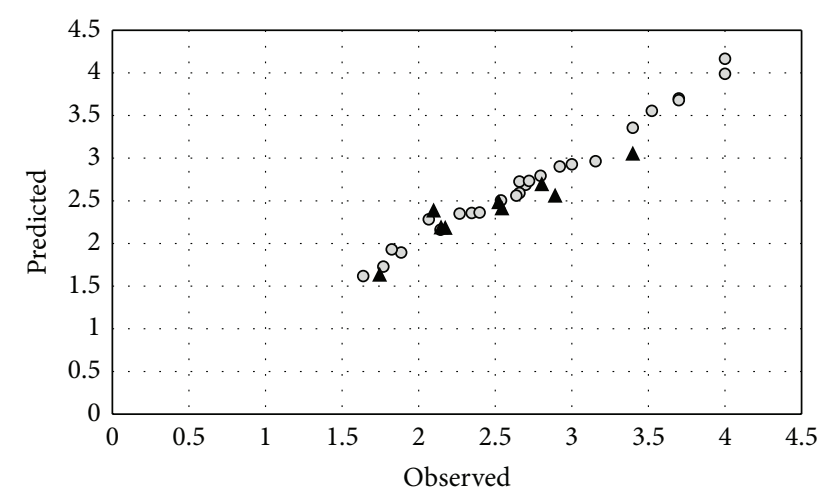

- Training set

^ Test set

FIGURE 2: Plot of observed versus predicted activity obtained from HQSAR model for training and test sets.

2.3. Prediction Set (Design of New Compounds). The prediction set contained 5 new not yet synthesized compounds having unknown observed values of activity against MMP12. They were designed based on the prediction ability of developed HQSAR model.

2.4. Molecular Docking. The molecular docking process was carried out employing Glide (Glide, version 5.7, Schrödinger, LLC, New York, NY, 2011) using default parameters. The protein (3F17) was prepared using Protein Preparation Wizard. Hydrogens were added, bond orders were assigned, overlapping hydrogens were corrected, missing side chains were added, and water molecules were removed. Finally, the protein structure was minimized by OPLS2005 force field. The prepared protein structure containing inhibitor molecule was used for active site definition (within $13 \mathrm{~A}$ from cocrystalized ligand). The 2D maps of ligands-receptor interactions were generated by ligand interaction diagram (Schrödinger molecular modeling suite).

\section{Results}

3.1. HQSAR Model Predictivity. The statistics for developed HQSAR model were shown in Table 2. The statistical parameters, $q^{2}, r^{2}$, SEE, and $r_{\text {pred }}^{2}$, showed the validity of our model. The best hologram model was generated using histogram length of 199 having six optimum components. Descriptors used for model generation were atoms, connections, and hydrogen atoms. The best generated model had crossvalidated $q^{2}$ of 0.697 and non-cross-validated $r^{2}$ value of 0.986 with a standard error of 0.93 . The total collection of the generated models for various histogram lengths comprises ensemble, and the ensemble value for $r^{2}$ was found to be 0.528 . The $Y$-randomization results indicated that the calculated $q^{2}(-1.238,-0.303,-0.793,0.081$, and -0.146$)$ and $r^{2}(0.683,0.088,0.086,0.241$, and 0.126$)$ for five random models are very low which also confirm the internal validity of the generated HQSAR model. The results of $r_{\text {pred }}^{2}$ calculation showed that the proposed HQSAR model was reliable

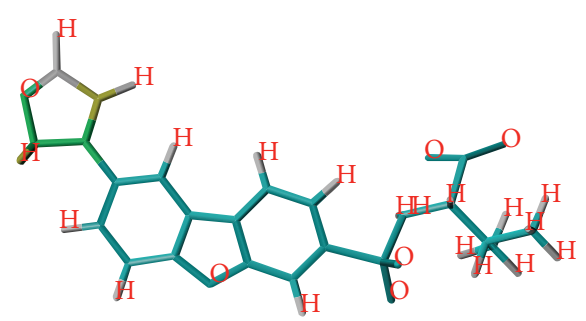

(a)

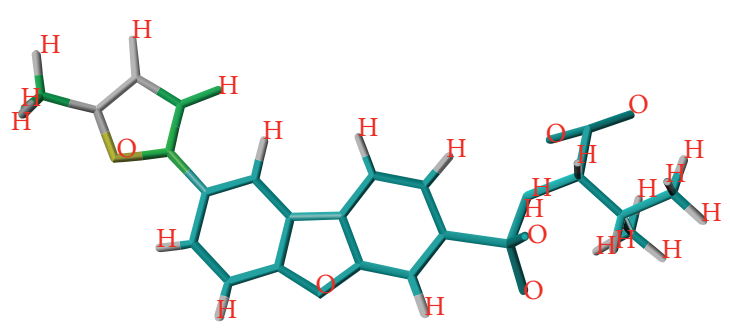

(b)

FIGURE 3: Contribution plot obtained from hologram quantitative structure activity relationship model for (a) compound 19 and (b) compound 20 .

and could successfully predict $\mathrm{pIC}_{50}$ for structurally related compounds which were not included in development of the models. The $r_{m}^{2}$ (after scaling) was 0.825 and delta $r_{m}^{2}$ was 0.107. Additionally, all four conditions of The GolbraikhTropsha method are satisfied $\left(r^{2}=0.876\right)$. Predicted values for the activity of molecules are shown in Table 1 and the experimental $\mathrm{pIC}_{50}$ against the values predicted by the HQSAR models are plotted (Figure 2).

3.2. HQSAR Atomic Contribution Plot. The generated model can be accessed through atomic contribution plot. The various colors of each atom correspond to various degrees of contribution towards the overall biological activity. Red, red orange, and orange depicted that the color belonging atoms were contributing negatively to the generated HQSAR model while colors reflecting yellow, green, and green blue were contributing positively to the model. Intermediate contributions were reflected by gray atom. The maximum common substructure was shown in cyan. Figure 3 depicts the contribution of the most potent compound 20 as well as compound 19.

3.3. Prediction Set (Design of New Virtual Compounds). This work allowed prediction of the activity of a set shown in Table 3 (not yet synthesized molecules). Their inhibitory activities were calculated according to the HQSAR model. They were designed based on compound 19 (one of the most active compounds). We had proposed a set of 5 new structures; some of them may show improved experimental MMP-12 inhibitory activity in comparison with the parent compound. This hypothesis and their selectivity should be verified experimentally.

3.4. Molecular Docking. The molecular docking approach was employed to further analyze the ability of designed 

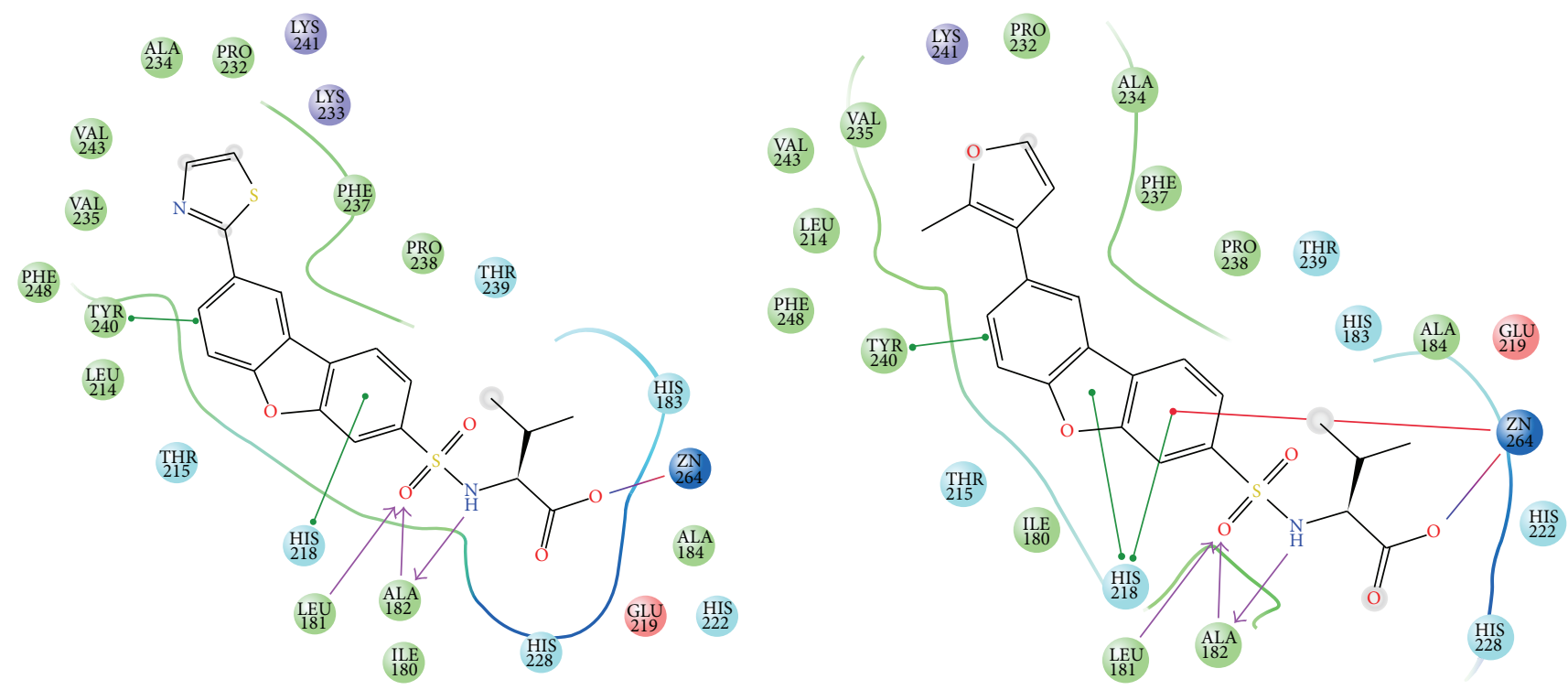

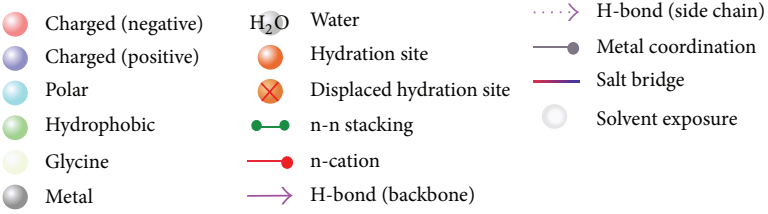

(a)

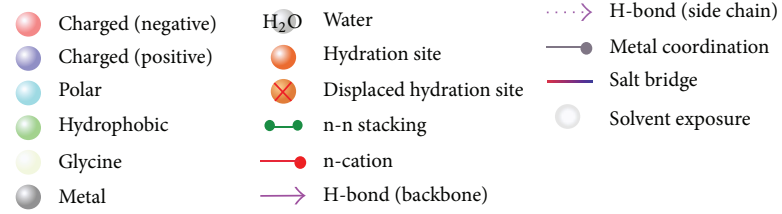

(b)
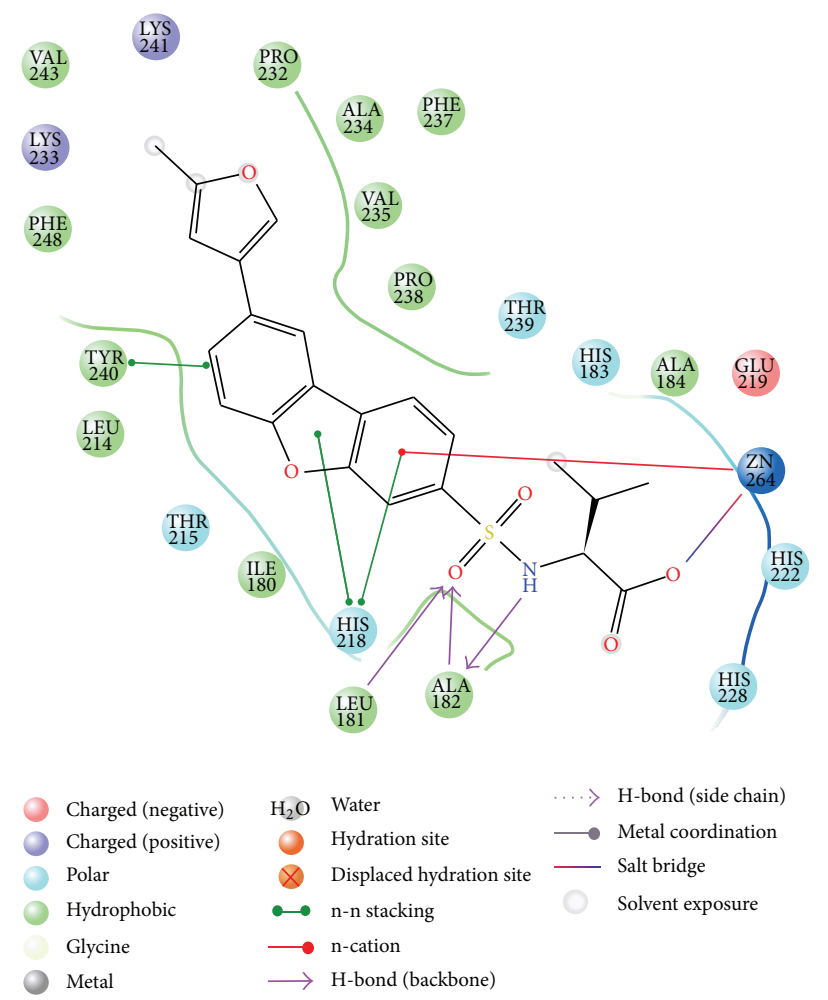

(c)

FIGURE 4: 2D interaction diagram of 3 docked designed compounds: (a) structure 26, (b) structure 20, and (c) structure n3. 


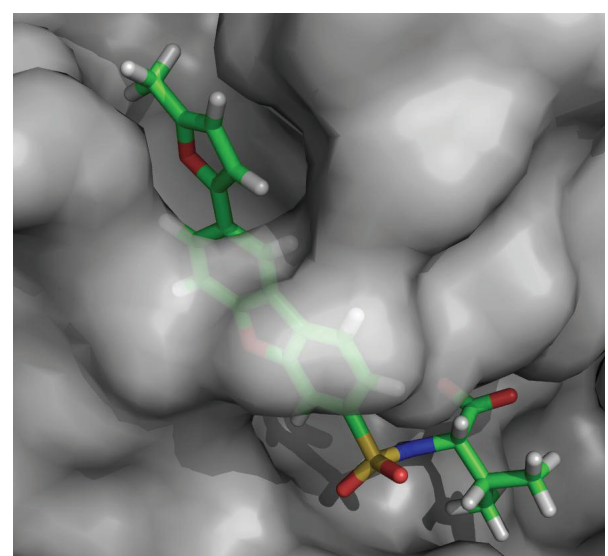

Figure 5: Compound 20 in the active site of MMP-12 (docked by Glide).

TABLE 2: Statistical characteristics of the developed HQSAR model.

\begin{tabular}{lc}
\hline Parameter & Value \\
\hline Number of compounds included in training set & 26 \\
Optimum number of components used in the PLS & 6 \\
analysis & 0.697 \\
$q^{2}$ (cross-validated correlation coefficient) & 0.428 \\
SEE $^{\mathrm{a}}\left(q^{2}\right)$ & 0.986 \\
$r^{2}$ (non-cross-validated correlation coefficient) & 0.093 \\
SEE $\left(r^{2}\right)$ & 0.528 \\
$r^{2}$ (ensemble $\left.{ }^{\mathrm{b}}\right)$ & 0.530 \\
SEE (ensemble) & 199 \\
Best hologram length & Atoms \\
& + Connections \\
Used information & + Hydrogen \\
& atoms \\
$r_{\text {pred }}^{2}$ & 0.8733 \\
\hline a SEE: standard error of estimate. ${ }^{b}$ For each hologram length, a model could \\
be established. The collection of these models comprises the ensemble.
\end{tabular}

compounds in inhibition of MMP-12. In Table 3, the docking scores of the 3 new designed compounds and 3 molecules from train set were reported. The binding positions of all new compounds were inspected for their binding conformation and interactions in MMP-12 active site. For n3, 2D diagram of ligand-receptor interaction was presented (Figure 4(c)). The various heterocyclic rings substituted on the dibenzofuran scaffold do not seem to have strong interactions with the binding pocket of MMP-12 as it was suggested previously by $\mathrm{X}$-ray crystallography [9]. However, if they have undesired properties they cannot fit in the narrow deep $\mathrm{Sl}^{\prime}$ pocket of MMP-12. On the other hand, they can induce steric hindrance that prevents other parts of the molecule to have strong interactions with residues in the binding pocket. The conformation of the heterocyclic ring upon ligand binding is demonstrated in Figure 5.

\section{Discussion}

We successfully developed a HQSAR model for prediction of some MMP-12 inhibitors with good internal and external validity. Subsequently, the model was used to predict the activity of new MMP-12 inhibitors. The binding energy of new not yet synthesized molecules was evaluated by molecular docking.

Crystal structures have provided useful information for developing selective inhibitors toward particular MMPs including MMP-12. The segment 241-245 of MMPs (MMP-1 numbering) has the highest sequence variability among the various MMP enzymes and could be a target for designing selective inhibitors. However, this segment is very flexible which makes the molecular modeling predictions using 3D structures of MMPs inaccurate [14, 15]. Only some small differences in the sizes of hydrophobic side chains were seen. For example, Val 235 in MMP-12 is replaced by Leu214 in MMP-13 which makes the MMP-13 binding pocket smaller and more hydrophobic. The series of MMP-12 inhibitors employed in this study had carboxylic acid zinc binding group. Changing the R group that was placed in hydrophobic pocket of MMP12 active site altered the potency and selectivity of these inhibitors. We modified this $\mathrm{R}$ group for fine tuning and designed new compound with promising MMP-12 inhibitory activity.

In the present study, we used HQSAR approach for a set of MMP-12 inhibitors. Contribution plot (Figure 3) showed that the green aromatic carbon was contributing positively to the model. Oxygen atom in furan ring was depicted by green or yellow and was contributing to the biological activity. Hydrogen molecules were rendered green, yellow, or white indicating that they showed intermediate contribution. The bulky group (methyl) was green indicating that it was contributing positively to the generated model, and it can be explained from the example that compound 20 was showing high potency. The developed model was used for the design of 5 new molecules. Overall docked conformation of the training set of inhibitors and new compounds in MMP-12 active site was similar to one determined by crystallography. Among new designed compounds, compounds n1, n2, and n3 had low SEP and their predicted activities were more reliable. Furthermore, compound $\mathrm{n} 3$ has the lowest docked energy.

\section{Conclusion}

In summary, we have developed a reliable HQSAR model for a series of tricycle cores containing MMP-12 inhibitors with dibenzofuran ring using activity data reported earlier $[9,10]$. We used HQSAR analysis to design new not yet synthesized potent MMP-12 inhibitors. Their binding energies were evaluated by docking studies but for further validation it needs synthesize of the proposed new compounds and subsequent enzyme inhibition study.

\section{Conflict of Interests}

The authors declare that there is no conflict of interests regarding the publication of this paper. 
TABLE 3: Predicted activities for the molecules based on the HQSAR model. Activities were shown as pIC ${ }_{50}(\mu \mathrm{M})$.

\begin{tabular}{|c|c|c|c|c|c|}
\hline Name & $\mathrm{R}$ & $\begin{array}{c}\text { Predicted } \\
\text { pIC }_{50} \text { values }\end{array}$ & SE of prediction & $\begin{array}{c}\text { Normalized mean } \\
\text { distance score }\end{array}$ & Docking score \\
\hline $\mathrm{n} 1$ & & 4.182 & 0.158579 & 0.161 & -12.038972 \\
\hline $\mathrm{n} 2$ & & 3.89 & 0.097741 & 0.076 & -13.92 \\
\hline n3 & & 3.942 & 0.093502 & 0.072 & -13.87 \\
\hline $\mathrm{n} 4$ & & 3.846 & 0.308627 & 0.291 & $-^{\mathrm{a}}$ \\
\hline n5 & & 4.003 & 0.325568 & 0.315 & $-^{\mathrm{a}}$ \\
\hline $20($ observed $=4)$ & & 4.032 & - & 0.043 & -13.7908 \\
\hline $19($ observed $=3.3979)$ & & 3.706 & - & 0.037 & -12.555956 \\
\hline $26($ observed $=3$ ) & & 2.945 & - & 0.009 & -11.781243 \\
\hline
\end{tabular}

${ }^{a}$ Docking was not performed for these compounds. 


\section{Acknowledgment}

This work has been partially supported financially by Sabzevar University of Medical Sciences.

\section{References}

[1] V. Vargová, M. Pytliak, and V. Mechírová, "Matrix metalloproteinases.", EXS, vol. 103, pp. 1-33, 2012.

[2] V. Lagente, C. Le Quement, and E. Boichot, "Macrophage metalloelastase (MMP-12) as a target for inflammatory respiratory diseases," Expert Opinion on Therapeutic Targets, vol. 13, no. 3, pp. 287-295, 2009.

[3] C. le Quément, I. Guénon, J.-Y. Gillon et al., "The selective MMP-12 inhibitor, AS111793 reduces airway inflammation in mice exposed to cigarette smoke," British Journal of Pharmacology, vol. 154, no. 6, pp. 1206-1215, 2008.

[4] P. Norman, "Selective MMP-12 inhibitors: WO-2008057254," Expert Opinion on Therapeutic Patents, vol. 19, no. 7, pp. 10291034, 2009.

[5] S. P. Gupta and V. M. Patil, "Specificity of binding with matrix metalloproteinases," EXS, vol. 103, pp. 35-56, 2012.

[6] K.-C. Tsai and T.-H. Lin, "A ligand-based molecular modeling study on some matrix metalloproteinase-1 inhibitors using several 3D QSAR techniques," Journal of Chemical Information and Computer Sciences, vol. 44, no. 5, pp. 1857-1871, 2004.

[7] B. Pirard, "Insight into the structural determinants for selective inhibition of matrix metalloproteinases," Drug Discovery Today, vol. 12, no. 15-16, pp. 640-646, 2007.

[8] HQSAR, Manual SYBYL-X 1.0, Tripos, St. Louis, Mo, USA, 2009.

[9] Y. Wu, J. Li, J. Wu et al., "Discovery of potent and selective matrix metalloprotease 12 inhibitors for the potential treatment of chronic obstructive pulmonary disease (COPD)," Bioorganic \& Medicinal Chemistry Letters, vol. 22, no. 1, pp. 138-143, 2012.

[10] L. Wei, L. Jianchang, W. Yuchuan et al., "A selective matrix metalloprotease 12 inhibitor for potential treatment of chronic obstructive pulmonary disease (COPD): discovery of (S)-2-(8(Methoxycarbonylamino)dibenzo $[b, d]$ furan-3-sulfonamido)3-methylbutanoic acid (MMP408)," Journal of Medicinal Chemistry, vol. 52, no. 7, pp. 1799-1802, 2009.

[11] K. Roy and I. Mitra, "On various metrics used for validation of predictive QSAR models with applications in virtual screening and focused library design," Combinatorial Chemistry and High Throughput Screening, vol. 14, no. 6, pp. 450-474, 2011.

[12] A. Golbraikh and A. Tropsha, "Beware of $q^{2}$ !," Journal of Molecular Graphics and Modelling, vol. 20, no. 4, pp. 269-276, 2002.

[13] K. Roy, P. Chakraborty, I. Mitra, P. K. Ojha, S. Kar, and R. N. Das, "Some case studies on application of "rm2" metrics for judging quality of quantitative structure-activity relationship predictions: emphasis on scaling of response data," Journal of Computational Chemistry, vol. 34, no. 12, pp. 1071-1082, 2013.

[14] P. Cuniasse, L. Devel, A. Makaritis et al., "Future challenges facing the development of specific active-site-directed synthetic inhibitors of MMPs," Biochimie, vol. 87, no. 3-4, pp. 393-402, 2005.

[15] J. A. Jacobsen, J. L. Major Jourden, M. T. Miller, and S. M. Cohen, "To bind zinc or not to bind zinc: an examination of innovative approaches to improved metalloproteinase inhibition," Biochimica et Biophysica Acta-Molecular Cell Research, vol. 1803, no. 1, pp. 72-94, 2010. 

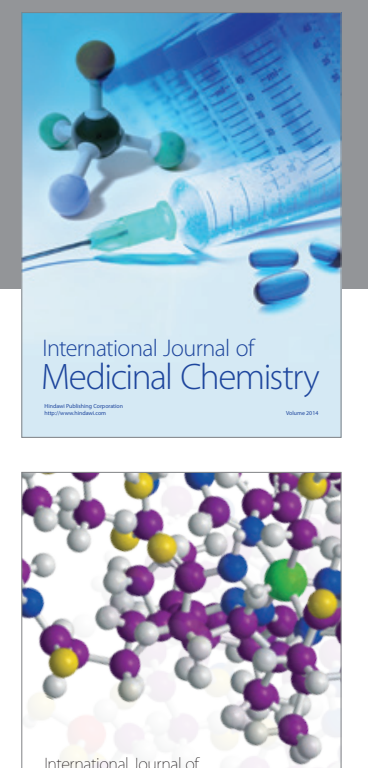

\section{Carbohydrate} Chemistry

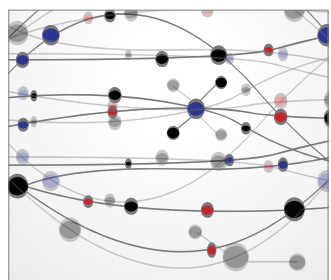

The Scientific World Journal
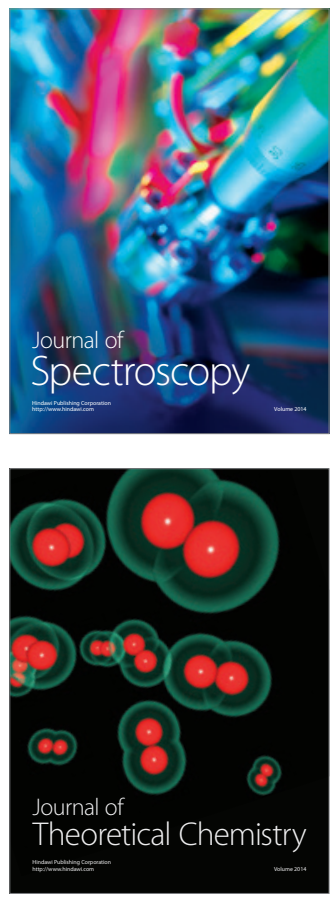
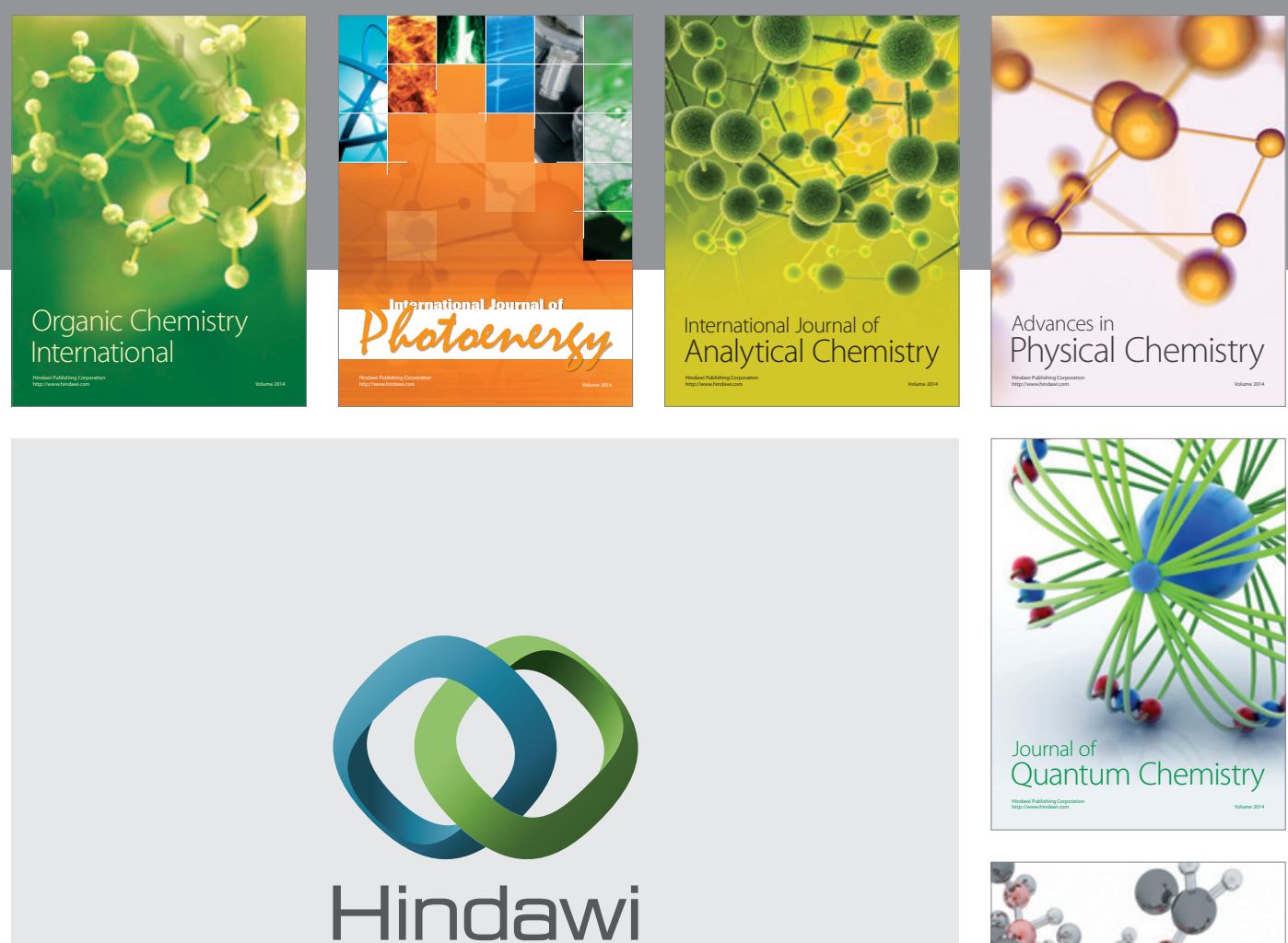

Submit your manuscripts at

http://www.hindawi.com

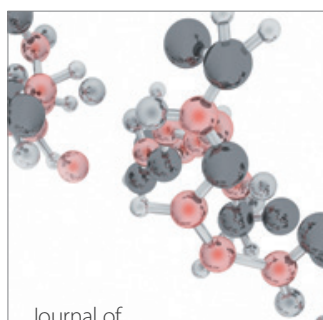

Analytical Methods

in Chemistry

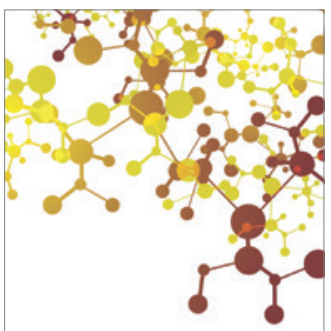

Journal of

Applied Chemistry

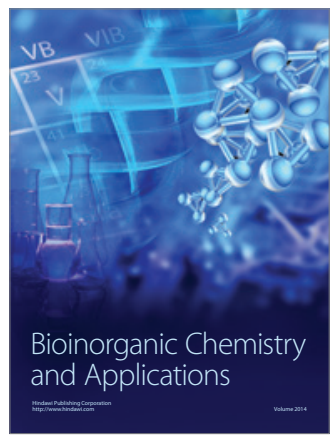

Inorganic Chemistry
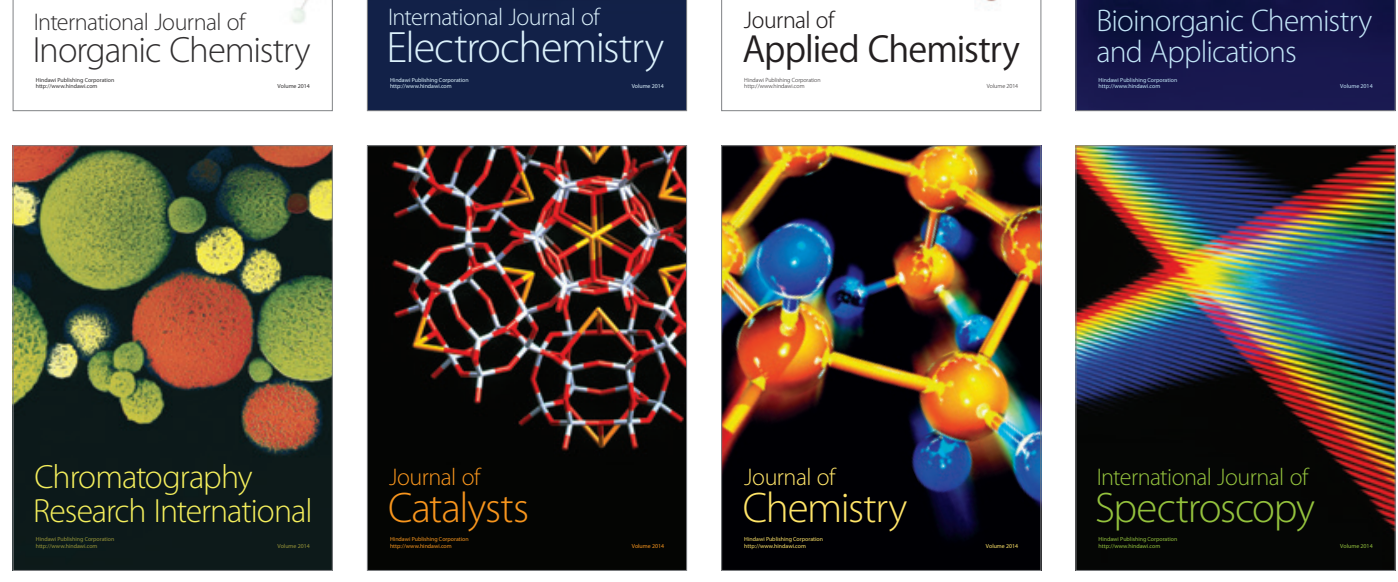